\title{
Performance Analysis of Different Watermarking Techniques on Medical Images
}

\author{
Geetamma Tummalapalli and Babji Prasad Chapa \\ Department of Electronics and Communication Engineering, GMRIT, Srikakulam, India \\ Corresponding author email: geethamma.t@gmrit.edu.in
}

\begin{abstract}
The protection of medical imaging data has recognized as a crucial problem in computer web safety. Digital Watermarking (DWM) has emerged as a potential method for medical content validation because it enables for the embedding of important information with the image, ensuring privacy, reliability, and validation by inserting a Digital Signature (DS) in the Medical image (MI). This work has focused on the performance study of Secure Medical Images utilizing Gaussian Filtering, LSB, DWT, and DWT-SVD image watermarking methods in this work. Various assaults are performed on the watermarked (WM) picture in order to assess the resilience of each method. Peak Signal to Noise Ratio (PSNR), Structural Similarity Index (SSIM), and Correlation coefficient for imperceptibility and resilience are used to assess the efficacy of the methods. In this article, several watermarking techniques such as Gaussian Filtering, LSB, DWT and DWT-SVD are developed and their benefits and drawbacks are discussed. Various assaults, such as rotation, cropping, and Gaussian noise, are conducted on the watermarked picture to test its resilience and imperceptibility. In terms of PSNR, SSIM, and NCC following assaults on watermarked images, DWT-SVD outperforms the other approaches. It may be concluded that different assaults for methods are conducted to evaluate watermarking systems, which can be selected for certain application areas.
\end{abstract}

KEY WORDS: DIGITAL WATERMARKING, GAUSSIAN FILTERING, DWT-SVD, PSNR, SSIM, CORRELATION COEFFICIENT.

\section{INTRODUCTION}

Medical image sharing via the internet is becoming more common for telediagnosis, telesurgery, and teleconsultation. Watermarking digital image (WMDI) is a comparatively modern technique in Hospital Information System (HIS) that is well adapted for medical and military purposes. The exponential evolution of digital technology has eased access to digital information. Digitization of multimedia data has allowed more reliable, quicker, and efficient digital data storage, transmission, and processing. It also has the unintended effect of resulting in the illicit creation and dissemination of digital material. Duplicating and modifying such digital data has become very simple and undetected.

Biosc Biotech Res Comm P-ISSN: 0974-6455 E-ISSN: 2321-4007

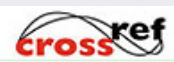

Identifiers and Pagination

Year: 2021 Vol: 14 No (6) Special Issue

Pages: $489-494$

This is an open access article under Creative
Commons License Attribn 4.0 Intl (CC-BY).
DOI: $h t t p: / / d x . d o i . o r g / 10.21786 / b b r c / 14.7 .100$

Commons License Attribn 4.0 Intl (CC-BY).
DOI: $h$ ttp://dx.doi.org/10.21786/bbrc/14.7.100
As a result of the huge development of computer networks that allow rapid and error-free transmission of any illegal duplicate and potentially modified copy of multimedia information, the danger of copyright infringement of multimedia data has risen.

Watermarking methods are divided into two types: spatial domain techniques and frequency domain approaches Manasha (2017). Watermarks are applied in the spatial domain by altering the pixel values of the source picture. It is simple to build in terms of computing, but it is vulnerable to a variety of assaults. Using transforms, the first picture is converted to a collection of frequency domain coefficients. Discrete Cosine Transform (DCT), Discrete Fourier Transform (DFT), and Discrete Wavelet Transform (DWT) are the transform domain methods utilized, and a watermark is applied to the modified coefficients. Significant bit, patchwork, and text mapping coding, among other things. Because traditional methods to content protection are no longer appropriate, it is essential to create new ways to enhance the security of medical pictures. The following factors are solely responsible for the security of medical

\section{Article Information}

Received: $15^{\text {th }}$ Aug 2021

Accepted after revision: $24^{\text {th }}$ Oct 2021 
pictures. i) Secrecy (ii) Integrity (iii) Authentication (Coatrieux, 2000; Coatrieux, 2006; Coatrieux, 2009).

Saeid-Fazli et al., (2010) examined the trade-off between LSB watermarking imperceptibility and resilience. This approach uses substantial bit planes of the WM image rather than the lower bit planes of the original image. Abdulla et al., (2011) presents a simple and resilient watermarking method that employs the 3rdand 4th LSB techniques. In concealing the data within the picture, the suggested method is more resilient than the conventional LSB technique. Two bits embed in the third and fourth LSBs using the suggested method. The experimental findings indicate that the watermarked picture has a better quality. Mohamed et al., (2011) introduced medical picture watermarking, which involves inserting a collection of data into a medical image. The watermarking technique uses the LSBs to verify the reliability and privacy of medical evidence and to preserve hospital and patient records confidentiality.

Hina et al., (2017) developed an alpha blending discrete wavelet transform method for embedding and extracting watermarks from original images. The findings demonstrate that the DWT method is resistant to a variety of typical image processing procedures. This method incorporates a visible watermark onto the cover picture. The extraction procedure necessitates the use of the cover picture. The scale parameters influence the quality of the recovered watermark image and the watermarked picture.

Watermarking method was implemented by Rita et al., (2016) using 2-level discrete wavelet transform. The variable perceptibility factor is utilized in this technique to introduce the WM into the low frequency component of the host picture. The simulation results of this technique indicate that the feature of the watermarked picture and the recovered WM are solely reliant on the visibility parameters, and that the 2-level DWT performs better than the 1-level discrete wavelet transform. Ramana Reddy et al. (2009) developed a method in which the WM is embedded and extracted in the frequency domain while also checking for salt and pepper and Gaussian noise assaults. They used a WM in the original image's DWT coefficients. Although the robustness of the watermarked picture improves, the quality of the final watermarked image decreases. Satish Chandra., Singular value decomposition (SVD) based digital watermarking method proposed to embed the visual and pseudorandom number sequence watermarks.

Watermarks were inserted in two levels in the original picture by Emir- Ganic et al., (2018) The first layer consisting of host picture and is splits into smaller blocks, after which the singular values of the watermark image are scaled by a configurable scaling factor and spread throughout the image blocks. In the second layer, all of the picture's singular values are scaled with a constant scaling factor and merged with the singular values of the watermarked image, and the resulting values are replaced with the original image singular values.

Aparna et. al. (2014) suggested watermarking methods based on DCT, DWT, and a mixture of the two. For embedding the watermark, these techniques use SVD of the cover picture and WM. This method is more resistant to shearing assaults, yet the watermark is more susceptible to noise attacks. Gaura et. al. (2009) technique segregates the the host picture using DWT. A reference image is created and stored for use during extraction. Both the reference picture and the watermark are subjected to SVD. The singular values of the reference picture are then changed using the watermark's singular values. In order to recreate the watermarked picture, inverse DWT is used. DWT converts a spatial picture to a frequency domain image by splitting it into four frequency sub bands: LL, HH, LH, and HL.

\section{MATERIAL ANID METHODS}

This paper preposes various spatial domain (LSB, Gaussian filtering method) \& frequency domain (DWT, DWT-SVD) based techniques for digital image watermarking analysis. Watermarked image subjected to various attacks and their impact is measured by calculating PSNR \& SSIM between the host image and the watermarked image. The Robustness is measured by calculating Normalized Correlation Coefficient (NCC) between the embedded watermark and extracted watermark image. The Gaussian filter employs a point spread function based on a two-dimensional distribution. The image is convolved with the 2D Gaussian distribution function to achieve this.

The Gaussian Kernel is separable, allowing for quick computing. Image brightness may be lost while using Gaussian filters. It does a better job of smoothing out photos. Its origins can be traced back to the human visual perception system. Gaussian filter is applied to the host with mask of $7 \times 7$ and the WM image to get corresponding high (Ihigh, Iwhigh) and low (Ilow, Iwlow) frequency components. Now low pass components of watermark image are embedded to low pass components of host image by using private key $\alpha$ as shown in Fig. 1, as the modifications done in the low frequency components will not affect the watermarked image. The following algorithm is used to embed the watermark into the lower components.

$$
\begin{aligned}
& \mathrm{MI}_{\text {bow }}=\mathrm{I}_{\text {fow }}+\left(\mathrm{I}_{\mathrm{w} 10 \mathrm{w}} / \alpha\right) \text { (1) } \\
& \mathrm{W}=\mathrm{I}_{\text {high }}+\mathrm{MI}_{\text {bow }}
\end{aligned}
$$

Where $\alpha$ is the visibility factor, MIlow is the modified low frequency components of host image, and $\mathrm{W}$ is watermarked image. During extraction, the watermarked image is filtered by the $7 \times 7$ Gaussian mask used in the embedding process to get Iewlow and Iewhigh. The lower components of host image areseparated from the lower components of watermarked image as shown in Fig. 2. The method used to extract the WM is.

$$
\mathrm{EW}=\left(\mathrm{I}_{\text {ow } 10 \mathrm{w}}-\mathrm{I}_{\mathrm{low}}\right) * \alpha
$$


Where $\alpha=0.6, \mathrm{I}_{\text {ewlow }}$ is the low frequency components of watermarked image, ${ }_{\mathrm{a}} \mathrm{nd}_{\text {lowis }}$ low frequency components of host image.

Figure 1: Block diagram of watermark embedding

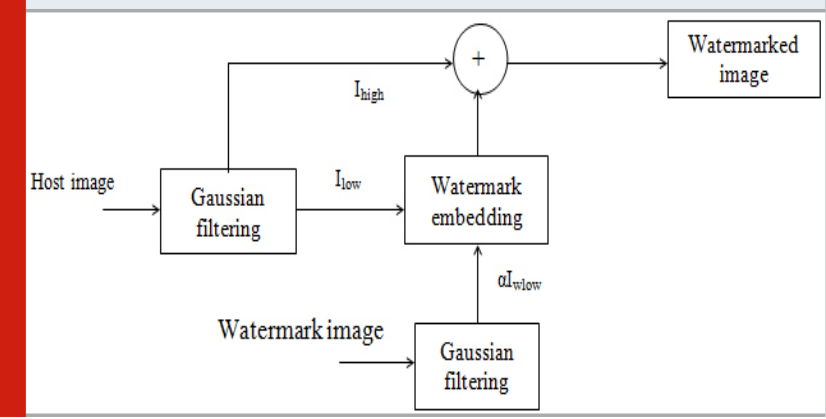

Figure 2: Block diagram of watermark extraction

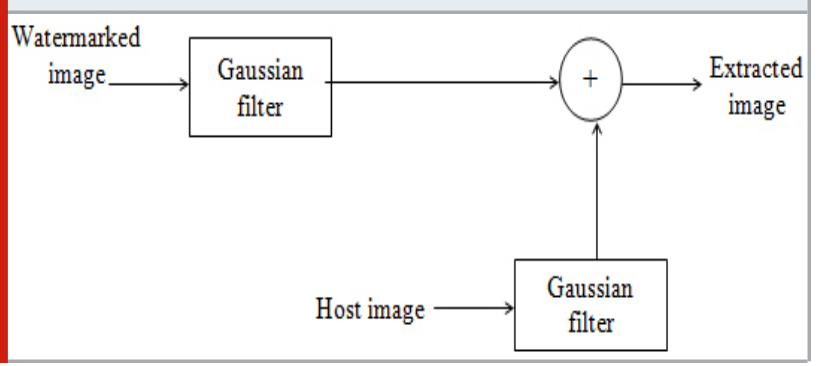

One of the Spatial domain methods is the LSB. In spatialdomain watermarking, information may be added by changing the values of the carrier signal's pixels. The Spatial-domain method directly modifies the colour values or intensities of certain chosen pixels. During this procedure, a watermark bit is inserted to each LSB pixel. The final bit of each pixel is read during the extraction or detection process to reveal the watermark information. The host and watermark pictures are first transformed to binary images. The host image's LSB plane is partitioned into $\mathrm{n}$ groups, with $\mathrm{n}$ equaling the number of watermark bits. The LSB plane is obtained and divided into $\mathrm{n}$ groups. The watermark bits are retrieved from each group by extracting the first bit. This method is vulnerable to a variety of assaults. It is very noise sensitive.

The host picture and the watermark image are decomposed into four sub-bandsLL, HL, LH, HH and WLL, WHL, WLH, WHH using first-level DWT.The majority of the energy is typically focused in the image's lower resolution area. As a result, the watermark is implanted in the lower half of the cover image, making the watermarked image more resilient without sacrificing image quality. So, the LL sub-band of WM is embedding into the LL sub-band of host image by using the below algorithm.

WMLL=LL+ (WLL. $\alpha)$

Where LL is low-frequency component of cover image, WLL is low frequency component of WM, $\alpha$ indicates visibility factor and WMLL is the watermarked image low frequency components. Similarly for 3 level DWT, single level DWT is performed on the cover image and LL sub band will be takenand 2 level DWT is performed on LL sub band to split into LL1, LH1, HL1, HH1 sub bands and same iscontinued for level 3 . After performing 3 Level DWT, embedding and extraction process are similar to 1 level DWT.The inverse DWT is used to approximate the watermarked image and other sub images of the cover image in order to acquire the watermarked image. Fig. 3 shows the WM embedding method.

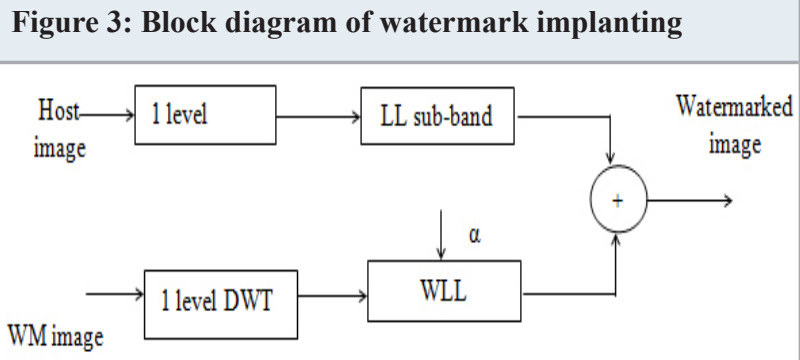

Figure 4: Block diagram of watermark extraction

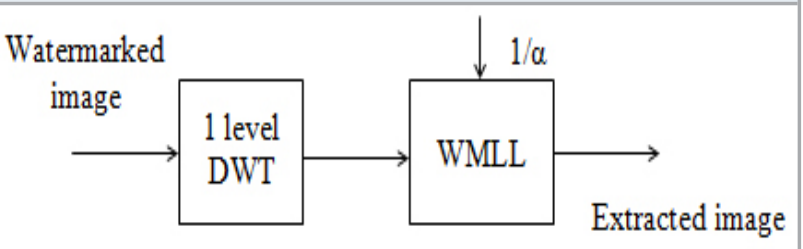

Figure 4.1: Block diagram of Watermark Extraction using SVD-DWT

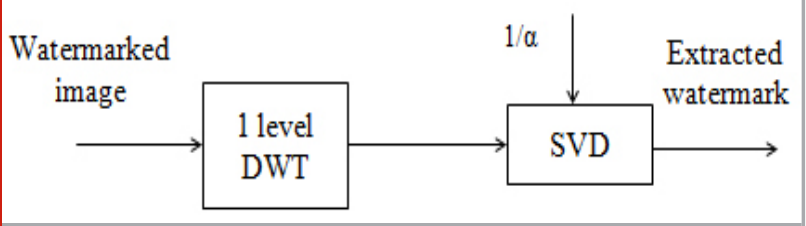

The watermarked image is decomposed into different frequency elements using DWT throughout the extraction procedure. From the approximate watermarked image, the WM image is extracted.

$\mathrm{EW}=(\mathrm{WMLL}-\mathrm{LL}) / \alpha(5)$

Where EW is the low frequency part of extracted watermark, WMLL is the low frequency part of the watermarked image, and LL is the low frequency element of WMI.After removal process, to obtain the final extracted WMI 1-level IDWT is applied. The watermark extraction process is shown in the below Fig. 4.

The singular matrices $\mathrm{U}, \mathrm{S}$, and $\mathrm{V}$ are produced by the SVD of an image. $\mathrm{U}$ and $\mathrm{V}$ are orthogonal matrices, while $\mathrm{S}$ is the diagonal matrix. As a decomposition matrix, SVD is particularly important in computer vision as it is an efficient tool for image processing. SVD of a cover imagecan be interpreted as [U S V] $=$ SVD Where $\mathrm{S}$ is the diagonal matrix $\&$ diagonal elements of S matrix comprises singular values; 
$\mathrm{S}$ values are non-negative numbers. $\mathrm{U}, \mathrm{V}$ is the orthogonal matrices UT U $=\mathrm{VT} \mathrm{V}=\mathrm{I}$ inverse of SVD can be taken as A $=U^{*} \mathrm{~S} * \mathrm{~V} 1$. First level DWT is operated on the host image to bifurcate into four sub bands LL, HL, LH, and HH. SVD is performed on LL to get diagonal and orthogonal values [Ui Si Vi] of host image. Also, SVD is performed on the watermark image to get diagonal and orthogonal values $\left[\mathrm{U}_{\mathrm{w}} \mathrm{S}_{\mathrm{w}} \mathrm{V}_{\mathrm{w}}\right.$ ]. Now diagonal matrix is modified by using the below equation and inverse SVD transform is applied to get the LL sub band.

Figure 5: Block Diagram of Watermark Embedding using SVD-DWT

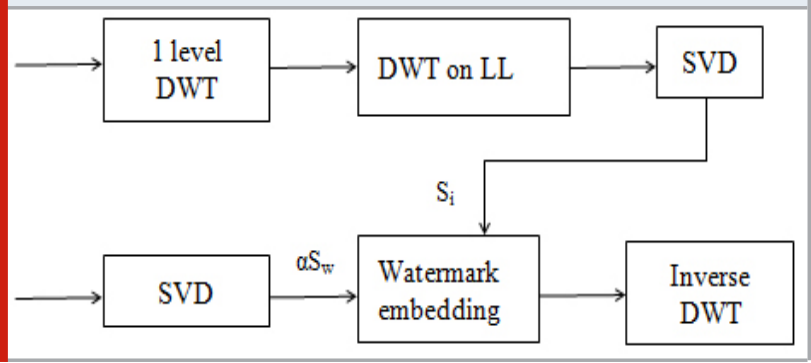

$\mathrm{S}_{\mathrm{WM}}=\mathrm{S}_{\mathrm{i}}+\mathrm{S}_{\mathrm{w}} * \alpha$

$\mathrm{MLL}=\mathrm{U}_{\mathrm{i}} \cdot \mathrm{S}_{\mathrm{WM}} \cdot \mathrm{V}_{\mathrm{i}}$

Where $\mathrm{Si}$ is the diagonal matrix of the host image and $\mathrm{Ui}$, $\mathrm{Vi}$ are the orthogonal matrices of the host image. Sw is the diagonal matrix of the watermark image and $\mathrm{U}_{\mathrm{w}}, \mathrm{V}_{\mathrm{w}}$ are the orthogonal matrices of the watermark image. And $\alpha$ is the visibility factor. SWM is the modified singular matrix. MLL is the modified LL sub band of the host image. To obtain the watermarked image inverse DWT is applied to approximate watermarked image and other sub images of host image. The following Fig. 5 shows the watermark embedding process.

In the extraction procedure, DWT is applied to the watermarked image, which decomposes the images into different frequency elements. SVD Transform is applied on the lower frequency sub band to get orthogonal and diagonal matrices [U S V]. Now watermark is extracted by using the following equation. Watermark extraction process is shown in the below Fig. 6 .

$\mathrm{Se}=(\mathrm{S}-\mathrm{Si}) / \alpha$

$\mathrm{EWM}=\mathrm{Uw}$. Se. Vw'

\section{RESULTS AND DISCUSSION}

In this section, performance of the different algorithms in reversible WM are compared among several imaging modalities viz.computed tomography(CT), magnetic resonance imaging (MRI), ultrasonic (US), endoscopic and angiographic images with respect to their PSNR and capacity rate (C). Here, 60 gray level medical images of different modalities such as MRI, CT and US have been kept in JPEG format with size of $512 \times 512 \times 8$ are considered for testing.DICOM standards are used tocapture the images. In Gaussian filtering method, to analyze the performance using Gaussian filter, watermark is embedded and extracted for different values of $\alpha$.In LSB method, watermark is extracted and to analyze the performance different attacks such as Gaussian noise, rotation, cropping are applied, watermark is embedded and extracted for different values of $\alpha$.

\begin{tabular}{|c|c|c|c|c|c|c|c|c|}
\hline \multirow{2}{*}{ S.NO } & \multirow{2}{*}{\multicolumn{2}{|c|}{$\alpha$}} & \multirow{2}{*}{ GAUSSIAN } & \multirow{2}{*}{ LSB } & \multicolumn{2}{|c|}{ DWT } & \multicolumn{2}{|c|}{ DWT-SVD } \\
\hline & & & & & L1 & L3 & L1 & L3 \\
\hline \multirow[t]{3}{*}{1} & \multirow[t]{3}{*}{$\begin{array}{l}0.0 \\
5\end{array}$} & PSNR & 34.4757 & \multirow[t]{3}{*}{-} & $\begin{array}{c}77.295 \\
7\end{array}$ & $\begin{array}{c}77.814 \\
1\end{array}$ & $\begin{array}{c}37.739 \\
6\end{array}$ & $\begin{array}{c}46.624 \\
6\end{array}$ \\
\hline & & SSIM & 0.9950 & & $\begin{array}{c}0.998 \\
5\end{array}$ & $\begin{array}{c}0.973 \\
0\end{array}$ & $\begin{array}{c}0.998 \\
5\end{array}$ & $\begin{array}{c}0.999 \\
8\end{array}$ \\
\hline & & $\mathrm{NCC}$ & 0.8808 & & 1 & 1 & $\begin{array}{c}0.999 \\
4\end{array}$ & $\begin{array}{c}0.954 \\
2\end{array}$ \\
\hline \multirow[t]{3}{*}{2} & \multirow[t]{3}{*}{0.4} & PSNR & 16.9721 & \multirow[t]{3}{*}{-} & $\begin{array}{c}59.23 \\
39\end{array}$ & $\begin{array}{c}59.752 \\
3 \\
\end{array}$ & $\begin{array}{c}19.738 \\
6\end{array}$ & $\begin{array}{c}28.786 \\
4\end{array}$ \\
\hline & & SSIM & 0.9041 & & $\begin{array}{c}0.929 \\
6\end{array}$ & $\begin{array}{c}0.770 \\
2\end{array}$ & $\begin{array}{c}0.966 \\
1\end{array}$ & $\begin{array}{c}0.993 \\
2\end{array}$ \\
\hline & & NCC & 0.9557 & & 1 & 1 & $\begin{array}{c}0.999 \\
8\end{array}$ & $\begin{array}{c}0.999 \\
7\end{array}$ \\
\hline \multirow[t]{3}{*}{3} & \multirow[t]{3}{*}{0.9} & PSNR & 11.0900 & \multirow[t]{3}{*}{-} & $\begin{array}{c}52.19 \\
03\end{array}$ & $\begin{array}{c}52.708 \\
6\end{array}$ & $\begin{array}{c}13.930 \\
8\end{array}$ & $\begin{array}{c}21.734 \\
5\end{array}$ \\
\hline & & SSIM & 0.6848 & & $\begin{array}{c}0.776 \\
4\end{array}$ & $\begin{array}{c}0.593 \\
6\end{array}$ & $\begin{array}{c}0.824 \\
8\end{array}$ & 0.9716 \\
\hline & & $\mathrm{NCC}$ & 0.5800 & & 1 & 1 & $\begin{array}{c}0.983 \\
8\end{array}$ & $\begin{array}{c}0.999 \\
9\end{array}$ \\
\hline
\end{tabular}


Table 2. Results after attacking the Watermarked image (PSNR Values in dB)

\begin{tabular}{|l|c|c|c|c|c|c|c|}
\hline \multirow{2}{*}{ S.NO } & \multirow{2}{*}{ ATTACKS } & \multirow{2}{*}{ GAUSSIAN } & \multirow{2}{*}{ LSB } & \multicolumn{2}{|c|}{ DWT } & \multicolumn{2}{|c|}{ DWT-SVD } \\
\cline { 5 - 8 } & & & & L1 & L3 & L1 & L3 \\
\hline 1 & No & 16.9721 & 78.6446 & 59.1903 & 59.7523 & 19.7164 & 28.7864 \\
\hline 2 & Gaussian noise & 15.2199 & 19.9821 & 69.3720 & 67.2205 & 16.7558 & 19.2301 \\
\hline 3 & Rotation & 8.9773 & 9.7924 & 50.8743 & 51.1806 & 9.2681 & 9.7065 \\
\hline 4 & Croping & 11.0375 & 13.2564 & 48.2005 & 51.3284 & 11.7462 & 13.0548 \\
\hline
\end{tabular}

Table 3. Results after attacking the Watermarked image (SSIM)

\begin{tabular}{|l|c|l|l|c|c|c|c|}
\hline \multirow{2}{*}{ S.NO } & \multirow{2}{*}{ ATTACKS } & \multirow{2}{*}{ GAUSSIAN } & \multirow{2}{*}{ LSB } & \multicolumn{2}{|c|}{ DWT } & \multicolumn{2}{|c|}{ DWT-SVD } \\
\cline { 4 - 8 } & & & & L1 & L3 & L1 & L3 \\
\hline 1 & No & 0.9041 & 1 & 0.9296 & 0.9102 & 0.9661 & 0.9932 \\
\hline 2 & Gaussian noise & 0.3266 & 0.3178 & 0.4986 & 0.5028 & 0.3325 & 0.3166 \\
\hline 3 & Rotation & 0.0225 & 0.0257 & 0.18126 & 0.1696 & 0.0253 & 0.0266 \\
\hline 4 & Croping & 0.0490 & 0.0550 & 0.1982 & 0.2002 & 0.0569 & 0.0561 \\
\hline
\end{tabular}

Table 4. Results after attacking the Watermarked image (NCC)

\begin{tabular}{|l|c|c|c|c|c|c|c|}
\hline \multirow{2}{*}{ S.NO } & \multirow{2}{*}{ ATTACKS } & \multirow{2}{*}{ GAUSSIAN } & \multirow{2}{*}{ LSB } & \multicolumn{2}{|c|}{ DWT } & \multicolumn{2}{|c|}{ DWT-SVD } \\
\cline { 4 - 8 } & & & & L1 & L3 & L1 & L3 \\
\hline 1. & No & 0.9908 & 1 & 1 & 1 & 0.9998 & 0.9997 \\
\hline 2. & Gaussian noise & 0.6835 & -0.0873 & -0.7085 & -0.7364 & 0.9149 & 0.9359 \\
\hline 3. & Rotation & -0.2352 & 0.0092 & -0.4608 & -0.4214 & 0.6634 & 0.0960 \\
\hline 4. & Croping & -0.3301 & -0.0265 & -0.1864 & -0.2593 & 0.9775 & 0.8722 \\
\hline
\end{tabular}

In order to increase the performance, the watermark is embedded in 1 and 3 levels in DWT andto analyze the performance different attacks such as Gaussian noise, rotation, cropping are applied. To analyze the performance of image watermarking using DWT-SVD, watermark is embedded and extracted for different values of $\alpha$. To increase the performance of watermarking, WM is embedded in DWT-SVD Level 1 and Level 3. The watermark is extracted by applying different attacks (Gaussian noise, rotation, cropping). The quality of watermarked image of Gaussian mask method, LSB, DWT and SVD-DWT methods is studied by calculating SSIM, PSNR and NCC.

The values of PSNR, SSIM and NCC for different values of $\alpha$ are observed. As $\alpha$ increases, PSNR and SSIM of these methods decreases, i.e., imperceptibility decreases. At the same time, NCC is increasing which results in increasing robustness. Comparatively $\alpha=0.4$ gives better imperceptibility and robustness. So, various attacks are applied for these methods at $\alpha=0.4$. The attacks applied are rotation $\left(10^{\circ}\right)$ in clockwise, cropping, and noise. Experimental results are shown in the table 2, 3 and 4.

For Method 1(Image Watermarking using Gaussian Filtering), the change in PSNR values is not much recognizable even with attacks but the quality of watermarked image is poor. It has less PSNR compared to the remaining three methods resulting in low imperceptibility. For Method 2 (Image watermarking using LSB), Imperceptibility is good without any attack but the quality of the watermarked image is getting degraded when the attacks are applied.

This technique is not very robust against various attacks and extremely sensitive to noise. For Method 3(Image watermarking using DWT), it has good quality of watermarked image compared to other methods i.e., imperceptibility is appreciable. It is less robust in nature againstthe attacks and also when compared to DWT-SVD Method. For Method 4(Image watermarking using DWTSVD), the quality of watermarked image is poor when compared to DWT method but it is much robust to various attacks than other methods.

\section{CONCLUSION}

Medical content authentication is critical when communicating medical pictures over the internet. This article examines a medical picture security system that uses several watermarking methods to provide authentication, dependability, and integrity. In this article, several watermarking techniques such as Gaussian Filtering, LSB, DWT (Level $1 \&$ amp: 3) and DWT-SVD (Level $1 \&$ amp: 
3) are developed and their benefits and drawbacks are discussed. Various assaults, such as rotation, cropping, and Gaussian noise, are conducted on the watermarked picture to test its resilience and imperceptibility. In terms of PSNR, SSIM, and NCC following assaults on watermarked images, DWT-SVD outperforms the other approaches. It may be concluded that different assaults for methods are conducted to evaluate watermarking systems, which can be selected for certain application areas.

\section{REFERENCES}

Abdullah Bamatraf, Rosziati Ibrahim, Mohd Najib, B. Mohd Salleh (2011). Digital watermarking algorithm using LSB, International Conference on Computer Applications and Industrial Electronics, pp. 155-159.

Aparna J R, Sonal Ayyappan (2009). Comparison of Digital Watermarking Techniques," International Conference for Convergence of Technology, IEEE 2014, pp. 1-6.

Bhatnagar, G. and Raman, B., A new robust reference watermarking scheme based on DWT-SVD. Computer Standards \& Interfaces, 31(5), pp.1002-1013.

Coatrieux, G., Maître, H., Sankur, B., Rolland, Y. and Collorec, R., (2000). Relevance of watermarking in medical imaging. In Proceedings 2000 IEEE EMBS International Conference on Information Technology Applications in Biomedicine. ITAB-ITIS 2000. Joint Meeting Third IEEE EMBS International Conference on Information Technol (pp. 250-255). IEEE.

Coatrieux, G., Lecornu, L., Sankur, B. and Roux, C., (2006). A review of image watermarking applications in healthcare. In 2006 International conference of the IEEE Engineering in Medicine and Biology Society (pp. 46914694). IEEE.

Coatrieux, G., Le Guillou, C., Cauvin, J.M. and Roux, C., (2008). Reversible watermarking for knowledge digest embedding and reliability control in medical images. IEEE Transactions on Information Technology in Biomedicine, 13(2), pp.158-165.
Chandra, D.S., (2002). Digital image watermarking using singular value decomposition. In The 2002 45th Midwest Symposium on Circuits and Systems, 2002. MWSCAS2002. (Vol. 3, pp. III-III). IEEE.

Choudhary, R. and Parmar, G., (2016). A robust image watermarking technique using 2-level discrete wavelet transform (DWT). In 2016 2nd International Conference on Communication Control and Intelligent Systems (CCIS) (pp. 120-124). IEEE.

Fazli, S. and Khodaverdi, G., (2009). Trade-off between imperceptibility and robustness of LSB watermarking using SSIM quality metrics. In 2009 Second International Conference on Machine Vision (pp. 101-104). IEEE.

D. M. Gaikwad, A. R. Karwankar (2016). A Review on Image Watermarking Techniques and Performance Measures, Journal of Information, Knowledge and Research in Electronics and Communication Engineering, 4(1), pp. 1302-1306.

Ganic, E., Zubair, N. and Eskicioglu, A.M., (2003). An optimal watermarking scheme based on singular value decomposition. In Proceedings of the IASTED international conference on communication, network, and information security, pp. 11-18.

Hajjaji, M.A., Mtibaa, A. and Bourennane, E.B., (2011). A Watermarking of Medical Image: Method Based" LSB". International Journal of Computer Science Issues.

Hina Lala (2017). Digital Image Watermarking using Discrete Wavelet Transform International Research Journal of Engineering and Technology, 4(1) pp. 1682-1685.

Reddy, P.R., Prasad, M.V. and Rao, D.S., (2009). Robust digital watermarking of color images under noise attacks. International Journal of Recent Trends in Engineering, 1(1), p.334.

Saqib, M. and Naaz, S., (2017). Spatial and frequency domain digital image watermarking techniques for copyright protection. Int. J. Eng. Sci. Technol. (IJEST), 9(2), pp.691-699. 